\title{
Prevalência de Fear of Missing Out em estudantes de uma instituição de ensino superior privada do Norte de Minas Gerais: comportamento e bem- estar digital no cotidiano universitário
}

\author{
Prevalence of Fear of Missing Out amongst students from a private higher \\ education institution from North of Minas Gerais, Brazil: digital behavior \\ and welfare in the university quotidian
}

\section{Prevalencia del Fear of Missing Out entre los estudiantes de una institución privada de educación superior en el Norte de Minas Gerais, Brasil: comportamiento y bienestar digital en la vida universitaria}

\author{
Beatriz Ferraz Pêgo ${ }^{1, a}$ \\ biaferrazpego@gmail.com | https://orcid.org/oooo-0002-3541-9384 \\ Beatriz Calixto Lopes ${ }^{1, a}$ \\ biaacalixto@gmail.com | https://orcid.org/o000-0001-5971-6300 \\ Gustavo Souza Santos ${ }^{1, b}$ \\ gustavo.santos@professor.unifipmoc.edu.br | https://orcid.org/oooo-0002-9712-2690 \\ Josiane Santos Brant Rocha ${ }^{1,2, c}$ \\ josiane.rocha@professor.unifipmoc.edu.br | https://orcid.org/o000-0002-7317-3880 \\ ${ }^{1}$ Centro Universitário FIPMoc. Montes Claros, MG, Brasil. \\ 2 Universidade Estadual de Montes Claros. Montes Claros, MG, Brasil. \\ a Graduanda em Comunicação Social - Publicidade e Propaganda do Centro Universitário FIPMoc. \\ ${ }^{\mathrm{b}}$ Mestrado em Geografia pela Universidade Estadual de Montes Claros. \\ ' Doutorado em Ciências da Saúde pela Universidade de Brasília.
}

\section{RESUMO}

Este estudo avaliou a prevalência de Fear of Missing Out (FOMO) em universitários, associando sexo, estágio de conclusão de curso e tipo de mídia acessada em uma instituição privada. A condição de FOMO foi investigada por meio do instrumento Scale of Assessment of FOMO com a participação de 311 estudantes. A prevalência de alto FOMO foi de 59,2\%. No modelo final, as variáveis que se mostraram associadas à FOMO foram ser do sexo feminino, estar cursando a primeira metade do curso, sensação de FOMO quando não se checa mídias sociais, uso de WhatsApp mais de 5 vezes ao dia, uso de Messenger mais de 5 vezes ao dia, FOMO durante o trabalho e FOMO quando sozinho. As sensações e sentimentos associados ao FOMO sugerem influências na tomada de decisões em rotinas comportamentais diárias na produção da individualidade, identidade, no convívio e na interação social cotidiana.

Palavras-chave: Fear of Missing Out; Mídias sociais; Estudantes; Internet; Comportamento. 


\section{ABSTRACT}

This study evaluated the prevalence of Fear of Missing Out (FOMO) in university students, associating sex, course completion stage and type of media accessed in a private institution. The condition of FOMO was investigated using the Scale of Assessment of FOMO instrument with the participation of 311 students. The prevalence of high FOMO was 59.2\%. In the final model, the variables that were associated with FOMO were being female, attending the first half of the course, feeling of FOMO when not checking social media, using WhatsApp more than 5 times a day, using Messenger more than 5 times a day, FOMO during work and FOMO when alone. The sensations and feelings associated with FOMO suggest influences in decisionmaking in daily behavioral routines in the production of individuality, identity, in social interaction and in daily social interaction.

Keywords: Fear of Missing Out; Social media; Students; Internet; Behavior.

\section{RESUMEN}

Este estudio evaluó la prevalencia del miedo a perderse (FOMO) en estudiantes universitarios, asociando el sexo, la etapa de finalización del curso y el tipo de medios a los que se accede en una institución privada. La condición de FOMO se investigó utilizando la escala de evaluación del instrumento FOMO con la participación de 311 estudiantes. La prevalencia de FOMO alto fue del 59,2\%. En el modelo final, las variables que se asociaron con FOMO fueron ser mujeres, estar en la primera mitad del curso, sentir FOMO al no revisar las redes sociales, usar WhatsApp más de 5 veces al día, usar Messenger más 5 veces al día, FOMO durante el trabajo y FOMO cuando está solo. Las sensaciones y sentimientos asociados con FOMO sugieren influencias en la toma de decisiones en las rutinas conductuales diarias en la producción de individualidad, identidad, en la interacción social y en la interacción social diaria.

Palabras clave: Fear of Missing Out; Medios de comunicación sociales; Estudiantes; Internet; Conducta.

Contribuição dos autores:

Concepção e desenho do estudo: Gustavo Souza Santos e Josiane Santos Brant Rocha.

Aquisição, análise ou interpretação dos dados: Beatriz Ferraz Pêgo e Beatriz Calixto Lopes.

Redação do manuscrito: Beatriz Ferraz Pêgo, Beatriz Calixto Lopes e Gustavo Souza Santos.

Revisão crítica do conteúdo intelectual: Josiane Santos Brant Rocha e Gustavo Souza Santos.

Declaração de conflito de interesses: não há.

Fontes de financiamento: não há.

Considerações éticas: aprovado pelo Comitê de Ética em Pesquisa do Centro Universitário FIPMoc, parecer n 3.353.373.

Agradecimentos/Contribuições adicionais: Ao Centro Universitário FIPMoc (UNIFIPMoc) pelo incentivo ao trabalho.

Histórico do artigo: submetido: 27 abr. 2020 | aceito: 23 set. 2020 | publicado: 22 mar. 2020.

Apresentação anterior: não há.

Licença CC BY-NC atribuição não comercial. Com essa licença é permitido acessar, baixar (download), copiar, imprimir, compartilhar, reutilizar e distribuir os artigos, desde que para uso não comercial e com a citação da fonte, conferindo os devidos créditos de autoria e menção à Reciis. Nesses casos, nenhuma permissão é necessária por parte dos autores ou dos editores. 


\section{INTRODUÇÃO}

Em tempos de cibercultura, os processos cotidianos são perpassados pela mediação de dispositivos comunicacionais (CASTELLS, 2006; LEMOS, 2018; JENKINS, 2009; HAN, 2018; BRAGA; 2020). A realidade acompanha um cenário em que a tecnologia se integra à quotidianidade, ocasionando uma hibridização entre a técnica e matriz humana (LIPOVETSKY, 2013; BAUMAN, 2001).

O relacionamento estabelecido entre sujeitos e a tecnologia não ocorre de maneira inerte ou dependente exclusivamente da necessidade e solução utilitária que códigos, linguagens e apetrechos fornecem. Ao contrário, a experiência tecnológica leva os sujeitos a novas configurações de vida, reorganizando padrões e vertendo sua existência em uma dimensão de sinergia humana e tecnológica (BAUDRILLARD, 2001; BAUDRILLARD, 2011).

Desse modo, embora sejam percebidas flexões na forma como se estabelece a sociabilidade on-line, esta reflete as características tipificadas da sociabilidade off-line. Um exemplo desta análise é a pulsão humana de estabelecer coletividades e desenvolver sentidos de pertencimento a grupos diversos e, neles e por meio deles, verter sua existência e identidade (PRZYBYLSKI; MURAYAMA; DEHAAN; GLADWELL, 2013).

Nesse sentido, as relações coletivas importam ou carregam relevância estrita para o desenvolvimento da performance individual. Isto é, as atividades dos membros dos diferentes grupos dos quais cada indivíduo faz parte são altamente consideradas por ele para seu desenvolvimento pessoal.

Nas múltiplas ambiências digitais, essas tessituras têm se desenvolvido consideravelmente. Diversos grupos e coletividades surgem e, com elas, modalidades de participação e interação, utilizando linguagens extrassimbólicas e que ampliam as relações. Além disso, em perspectiva está a disjunção espaçotemporal da forma e do conteúdo das relações.

Tem sido percebido, tanto socialmente quanto a partir de estudos (ALT, 2018; HETZ; DAWSON; CULLEN, 2015; CASTELLS, 2013; ABEL; BUFF; BURR, 2016), um sentimento de avidez por tomar conhecimento e ter contato com o que os membros dos diversos grupos dos quais os sujeitos na esteira on-line fazem parte. Tal avidez causa instância, ansiedade e comportamentos depressivos. Este fenômeno foi nomeado de Fear of Missing Out (FOMO).

O FOMO designa um sentimento ou sensação de difícil associação e, por vezes, avassaladora, de um indivíduo que percebe que está perdendo algo relacionado ao que seus pares estão fazendo, conhecendo, possuindo e vivendo e que o coloca em posição inferior (ABEL; BUFF; BURR, 2016).

Nota-se que o FOMO designa um quadro complexo de relações de sociabilidade e performance pessoal - com flexões comportamentais - e que necessitam ser avaliados em determinados grupos, a fim de se conhecer a dinâmica das relações e como elas modulam as experiências cotidianas.

Desse modo, o presente estudo tem como objetivo avaliar a prevalência de Fear of Missing Out (FOMO) associado a sexo, estágio de conclusão de curso e tipo de mídia acessada em estudantes universitários de uma instituição privada.

\section{METODOLOGIA}

Trata-se de um estudo transversal e analítico, realizado na cidade de Montes Claros/MG, Brasil, no período de agosto a outubro de 2019, cuja população foi composta por 5.000 estudantes dos cursos de graduação de uma IES privada que atende a região Norte do estado de Minas Gerais e o Sul baiano.

A escolha do campo de investigação vinculado a uma IES privada em Montes Claros se dá por suas características de centralidade socioeconômica e espacial no Norte do estado mineiro. A cidade congrega serviços, fluxos e equipamentos públicos arrolando o desenvolvimento regional, marcado por uma história de precarização socioeconômica e diversidade sociocultural, comunicando-se geográfica e economicamente 
com o sul baiano e as outras regiões do estado (VAUGHN, 2012; GONÇALVES; SILVEIRA; CAMPOS; COSTA, 2001).

A amostragem foi do tipo probabilístico. Os estudantes foram selecionados mediante sorteio feito a partir das listas de matriculados fornecidos pela instituição, cuja autorização foi obtida previamente. O contato foi intermediado por cada coordenação de curso, de modo que os estudantes fossem convidados para participar dos estudos, registrassem seu assentimento e, em caso de recusa, outro estudante pudesse ser convidado por meio de novo sorteio.

Os estudantes foram divididos por 16 grupos, considerando o número de cursos de graduação em vigência, para que se obtivesse representantes do total discente. Os critérios de seleção incluíram possuir a matrícula ativa e regular na instituição lócus do estudo e ter algum acesso regular a mídias sociais, sem restrições sociodemográficas ou de outra natureza, uma vez que a dimensão do FOMO foi o foco da investigação.

A coleta de dados foi realizada durante os intervalos regulares de aulas, de modo a não prejudicar a rotina acadêmica. No momento da aplicação do questionário, os estudantes eram instruídos previamente sobre os trâmites da pesquisa, assinando o Termo de Consentimento Livre e Esclarecido e o Consentimento de Pós-informação.

Para avaliação de FOMO, os estudantes responderam ao questionário validado (ABEL; BUFF; BURR, 2016), o Scale of Assessment of FOMO, cujas variáveis incluem cinco dimensões da experiência pessoal de FOMO a partir da necessidade de checar redes sociais quando se está na companhia de outras pessoas, quando não se pode acessá-las por algum motivo, quando se está em atividades de trabalho, quando se está em atividades de estudo ou sozinho. As variáveis foram avaliadas em uma escala de 1 a 7 , sendo 1 a percepção muito fraca e 7, muito forte.

O usufruto da Scale of Assessment of FOMO parte do pressuposto de que o FOMO se desencadeia como uma pulsão sobre a experiência cotidiana dos sujeitos, de modo intra e intersubjetivo. Visto que o FOMO se expressa por meio de uma avidez instável e uma sensação de perda da atualidade, a autopercepção aferida pela escala permite o diálogo com a percepção de intensidade confessional pelos sujeitos, visto que sentir FOMO é um processo de intensidades variadas, mensuradas pelos indivíduos pelo quão pungente sua ocorrência se estabelece.

Adicionalmente, para fins de associação entre variáveis, foram analisádos os dados sociodemográficos como sexo (masculino ou feminino), idade, estágio de conclusão do curso de graduação (primeira ou segunda metade) e frequência de usos de serviços de mídias sociais com a estimativa de acessos por dia (de 1 a mais de 10 vezes por dia) para cada plataforma investigada (Facebook, Instagram, Twitter, Messenger, WhatsApp e Telegram).

Os dados foram tabulados no programa estatístico Statistical Package for the Social Science (SPSS, versão 21, Chicago, Illinois). Inicialmente, foram realizadas análises descritivas de todas as variáveis investigadas por meio de suas distribuições de frequências e, posteriormente, foram desenvolvidas análises bivariadas da variável desfecho com cada variável independente, utilizando-se o Teste Qui-Quadrado. A proposta do teste é comparar proporções de regularidades e suas prováveis divergências entre um evento e sua frequência.

Foram estimadas Razões de Prevalência (RP) brutas, com seus respectivos intervalos de $95 \%$ de confiança. As variáveis que apresentaram nível descritivo (valor-p) inferior a 0,25 foram selecionadas para análise múltipla que utilizou o modelo de regressão de Poisson hierarquizado, adaptado ao modelo proposto por outros autores (JACKSON; ERVIN; GARDNER; SCHMITT, 2001).

A percepção de FOMO foi classificada, conforme o modelo utilizado, em alto e baixo FOMO. Foram estimadas razões de prevalências (RP) ajustadas com seus respectivos intervalos de 95\% de confiança, permanecendo no modelo somente aquelas que apresentaram nível descritivo $\mathrm{p}<0$,05. Em cada nível 
hierárquico, adotou-se o procedimento passo à frente (stewise forward procedure), ou seja, iniciou-se o modelo com a variável com maior significância estatística, selecionada na análise bivariada e, a seguir, foram acrescentadas as demais variáveis.

\section{RESULTADOS E DISCUSSÃO}

A amostra foi composta por 311 estudantes dos 16 cursos de graduação da IES investigada, com idade compreendida entre 18 e 34 anos, sendo que 59,2\% apresentaram alto FOMO (tabela 1).

\section{Tabela 1 - Razão de prevalência geral do grupo amostral}

\begin{tabular}{l|cccc} 
& $\mathbf{n}$ & $\%$ & \% válida & \% cumulativa \\
\hline Baixo & 127 & $40,8 \%$ & 40,8 & $40,8 \%$ \\
Alto & 184 & $59,2 \%$ & 59,2 & $100,0 \%$ \\
Total & 311 & $100,0 \%$ & 100,0 & \\
\hline
\end{tabular}

Fonte: elaborado pelos autores.

O resultado da análise bivariada revelou que as variáveis que se associaram com o desfecho de alto FOMO foram sexo $(\mathrm{p}=0,007)$, estar cursando a primeira metade do curso $(\mathrm{p}=\mathrm{o}, 000)$, sentir FOMO quando não checa redes sociais ( $\mathrm{p}=\mathrm{o}, \mathrm{OOO}$ ), FOMO mesmo quando as redes sociais são checadas $(\mathrm{p}=0,000)$, FOMO mesmo quando em companhia de outras pessoas ( $\mathrm{p}=0,013$ ), FOMO mesmo quando não se é possível acessar redes sociais $(\mathrm{p}=\mathrm{0}, 000)$, FOMO durante as aulas $(\mathrm{p}=0,000)$, quando se está sozinho $(\mathrm{p}=0,000)$ e no trabalho $(\mathrm{p}=\mathrm{o}, 000)$.

O desfecho de alto FOMO se associou ainda com o uso de redes sociais checadas mais do que 5 vezes por dia, como Facebook $(\mathrm{p}=0,128)$, WhatsApp $(\mathrm{p}=0,000)$, Messenger $(\mathrm{p}=0,003)$ e Telegram $(\mathrm{p}=0,162)$. Ressalta-se que houve uma elevada prevalência de FOMO em todas as variáveis independentes apresentadas (tabela 2). 
Tabela 2 - Caracterização da amostra e razão de prevalência (RP) bruta para FOMO associada ao uso de redes sociais

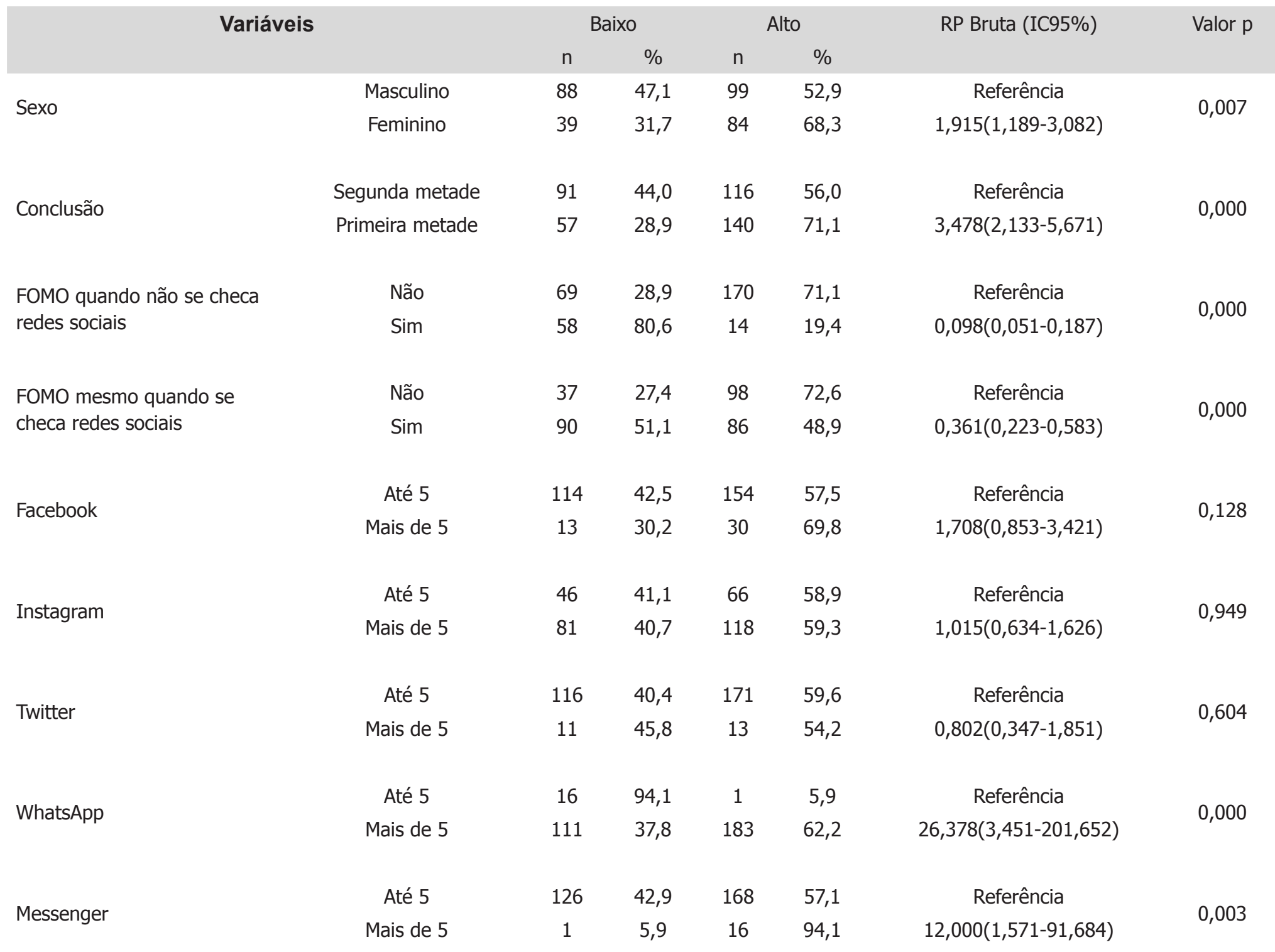




\begin{tabular}{|c|c|c|c|c|c|c|c|}
\hline \multicolumn{2}{|c|}{ Variáveis } & \multicolumn{2}{|c|}{ Baixo } & \multicolumn{2}{|c|}{ Alto } & \multirow[t]{2}{*}{ RP Bruta (IC95\%) } & \multirow[t]{2}{*}{ Valor $p$} \\
\hline & & $\mathrm{n}$ & $\%$ & $n$ & $\%$ & & \\
\hline \multirow{2}{*}{ Telegram } & Até 5 & 124 & 40,4 & 183 & 59,6 & Referência & \multirow{2}{*}{0,162} \\
\hline & Mais de 5 & 3 & 75,0 & 1 & 25,0 & $0,226(0,23-2,196)$ & \\
\hline \multirow{2}{*}{ FOMO quando acompanhado } & Às vezes & 111 & 44,2 & 140 & 55,8 & Referência & \multirow{2}{*}{0,013} \\
\hline & Sempre & 16 & 26,7 & 44 & 73,3 & $2,180(1,168-4,070)$ & \\
\hline \multirow{2}{*}{$\begin{array}{l}\text { FOMO quando não se pode } \\
\text { acessar redes sociais }\end{array}$} & Às vezes & 103 & 49,0 & 107 & 51,0 & Referência & \multirow{2}{*}{0,000} \\
\hline & Sempre & 24 & 23,8 & 77 & 76,2 & $3,088(1,814-5,258)$ & \\
\hline \multirow{2}{*}{ FOMO durante as aulas } & Às vezes & 97 & 55,4 & 78 & 44,6 & Referência & \multirow{2}{*}{0,000} \\
\hline & Sempre & 30 & 22,1 & 106 & 77,9 & $4,394(2,657-7,267)$ & \\
\hline \multirow{2}{*}{ FOMO durante o trabalho } & Às vezes & 111 & 53,6 & 96 & 46,4 & Referência & \multirow[t]{2}{*}{0,000} \\
\hline & Sempre & 16 & 15,4 & 88 & 84,6 & $6,359(3,495-11,572)$ & \\
\hline \multirow{2}{*}{$\begin{array}{l}\text { FOMO quando se está } \\
\text { sozinho }\end{array}$} & Às vezes & 53 & 80,3 & 13 & 19,7 & Referência & \multirow[t]{2}{*}{0,000} \\
\hline & Sempre & 74 & 30,2 & 171 & 69,8 & $9,421(4,845-18,321)$ & \\
\hline
\end{tabular}

Fonte: elaborado pelos autores.

As variáveis independentes que apresentaram associação com FOMO após análise multivariada foram ser do sexo feminino ( $\mathrm{RP}=1,85$; $\mathrm{p}=$ o,o40); estar na

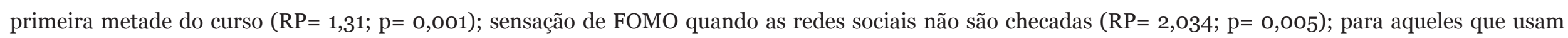

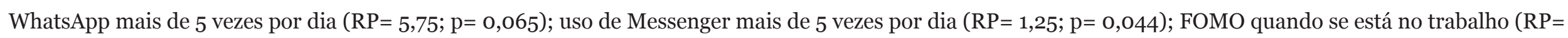
1,34; $\mathrm{p}=0,001)$; e FOMO quando se está sozinho $(\mathrm{RP}=1,76 ; \mathrm{p}=0,025)$ (Tabela 3). 
Tabela 3 - Razão de prevalência ajustada para FOMO de acordo com fatores sociodemográficos, uso de redes sociais e ocasião

\begin{tabular}{|c|c|c|c|}
\hline Variável & & $\begin{array}{l}\text { RP (IC95\%) } \\
\text { Ajustada }\end{array}$ & Valor $\mathbf{p}$ \\
\hline Sexo & $\begin{array}{l}\text { Feminino } \\
\text { Masculino }\end{array}$ & $\begin{array}{l}\text { 1,185(1,008-1,394) } \\
\text { Referência }\end{array}$ & 0,040 \\
\hline $\begin{array}{l}\text { Estágio de conclusão } \\
\text { do curso }\end{array}$ & $\begin{array}{l}\text { Primeira metade } \\
\text { Segunda metade }\end{array}$ & $\begin{array}{c}\text { 1,318(1,118-1,554) } \\
\text { Referência }\end{array}$ & 0,001 \\
\hline $\begin{array}{l}\text { Quando não checo redes } \\
\text { sociais, sinto que estou } \\
\text { perdendo algo }\end{array}$ & $\begin{array}{l}\text { Sim } \\
\text { Não }\end{array}$ & $\begin{array}{c}\text { 2,034(1,237-3,345) } \\
\text { Referência }\end{array}$ & 0,005 \\
\hline Uso de WhatsApp & $\begin{array}{l}\text { Mais de } 5 \text { vezes } \\
\text { Até } 5 \text { vezes }\end{array}$ & $\begin{array}{c}\text { 5,755(0,897-36,903) } \\
\text { Referência }\end{array}$ & 0,065 \\
\hline Uso de Messenger & $\begin{array}{l}\text { Mais de } 5 \text { vezes } \\
\text { Até } 5 \text { vezes }\end{array}$ & $\begin{array}{l}\text { 1,254(1,006-1,564) } \\
\text { Referência }\end{array}$ & 0,044 \\
\hline $\begin{array}{l}\text { Quando estou no trabalho, } \\
\text { sinto que estou perdendo algo }\end{array}$ & $\begin{array}{l}\text { Sempre } \\
\text { Às vezes }\end{array}$ & $\begin{array}{l}\text { 1,340(1,134-1,582) } \\
\text { Referência }\end{array}$ & 0,001 \\
\hline $\begin{array}{l}\text { Quando estou sozinho, } \\
\text { sinto que estou perdendo algo }\end{array}$ & $\begin{array}{l}\text { Sempre } \\
\text { Às vezes }\end{array}$ & $\begin{array}{c}\text { 1,768(1,074-2,912) } \\
\text { Referência }\end{array}$ & 0,025 \\
\hline
\end{tabular}

Fonte: elaborado pelos autores.

Em suma, ocorrências significativas para os indivíduos que apresentaram alto FOMO foram observadas especialmente em mulheres. Considerando a etapa de realização do curso superior, aqueles que estão na primeira metade da formação têm maior ocorrência do fenômeno. Outro momento em que o alto FOMO se manifesta é na variável de quando, por razões diversas, não se pode acessar ou checar as redes sociais, assim como durante o trabalho ou quando se está sozinho. Aqueles que acessam WhatsApp e Messenger mais de 5 vezes por dia também apresentam maior expressividade de alto FOMO.

\section{DISCUSSÃO}

Este estudo encontrou elevada prevalência de alto FOMO em acadêmicos matriculados na IES investigada com associação dos fatores ser do sexo feminino, estar na primeira metade do curso, sentir FOMO quando não se pode acessar mídias sociais, quando se está no trabalho e quando se está sozinho, além de sentir FOMO quando se usa WhatsApp e Messenger mais de 5 vezes por dia. Em geral, os achados revelaram que mais da metade dos estudantes apresenta alto FOMO.

O quadro de alto FOMO deriva desse quadro, no qual a pervasividade dos serviços, aplicativos, trocas de mensagens e fluxos de informações compõem o quadro situacional dos indivíduos, como um contexto de elaboração e realização da vida material e imaterial ${ }^{11,14}$. A ambiência on-line reflete a forma, o conteúdo e o contorno das relações sociais e da construção da subjetividade ${ }^{11}$. 
Algum grau de FOMO é percebido no cotidiano dos sujeitos, todavia os indicativos desse estudo e de outros estudos (HETZ; DAWSON; CULLEN, 2015; CASTELLS, 2013; MORAHAN-MARTIN, 2009; ABELL; BUGLASS; BETTS, 2019; CASALE; FIORAVANTI, 2020; COCO et al, 2020; KARGIN; POLAK; SIMSEK, 2020; FABRIS; MARENGO; LONGOBARDI; SETTANNI, 2020; LOPES; PÊGO; SANTOS, 2019; LOPES; SANTOS; PÊGO, 2019) implicam em uma prevalência de alto grau do fenômeno, imbricando em dinâmicas emocionais e comportamentais difusas, resvalando sobre a vida pessoal, o trabalho, os estudos, os relacionamentos e a produção do cotidiano intra e intersubjetivo.

A maior prevalência do fenômeno em mulheres parece sugerir implicações de gênero nas tratativas digitais (FALLOWS, 2005). Pode-se avaliar a discrepância de tipo e qualidade de acesso a internet por homens e mulheres, sobretudo observando ambientes hostis e estereotipados entre seus papéis e performances no meio digital. Esse aspecto pode ter aderência com o perfil feminino apresentando FOMO elevado, isto é, a partir da conjuntura desigual, das expectativas e experiências diante do feminino, comportamentos ansiosos e exacerbados podem ser caminhos de explicação e investigação futura.

Disparidades de uso, figuração e performance são perceptíveis nas ambiências on-line em relação ao primado masculino sobre as mulheres (MORAHAN-MARTIN, 2009; FALLOWS, 2005). Essa lacuna não diz respeito necessariamente ao acesso quantitativo e/ou qualitativo das ferramentas, mas às representações sociais e midiáticas da figura e dos papéis femininos que denotam pujança sobre as ambiências digitais (JACKSON; ERVIN; GARDNER; SCHMITT, 2001; MORAHAN-MARTIN, 2009; FALLOWS, 2005). Pressões sociais sobre perfis femininos ideais e multifacetados geram uma necessidade de adequação e validação das mulheres, o que pode ter reflexo nos comportamentos de consumo e compartilhamento de informações em rede (JACKSON; ERVIN; GARDNER; SCHMITT, 2001; MORAHAN-MARTIN, 2009).

Observou-se que os sujeitos cursando a primeira metade dos cursos apresentam maior prevalência de alto FOMO. Isto é, o grupo de sujeitos majoritariamente mais jovens. A faixa etária compreendida pelo estudo evoca sujeitos que têm nas mídias sociais a articulação de práticas pessoais e coletivas de produção do cotidiano e da subjetividade, seja por razões profissionais, recreativas ou objetivas em algum nível. Trata-se de gerações ou nativas digitais ou com acesso mais tenro aos meios, sendo naturalmente mais imersos em seus sistemas e dinâmicas.

Os resultados vão de encontro a estudos que denotam um prognóstico crescente de FOMO e outras variáveis situacionais da contemporaneidade como autoestima, depressão, ansiedade e ostracismo (ABEL; BUFF; BURR, 2016). Tais variáveis encontram ressonância com o fator idade, em que indivíduos mais jovens tendem a apresentar níveis elevados, inclusive de FOMO. O estudo demonstrou que estudantes na primeira metade dos cursos - a partir da amostra, notadamente mais jovens - apresentaram alto FOMO.

Nas variáveis de percepção de FOMO quando não se pode ter acesso às redes sociais e FOMO mesmo quando as redes sociais estão acessadas encontrou resultados significativos. No entanto, a sensação de que se está perdendo algo quando o acesso não é possível é ligeiramente menor, enquanto a percepção mesmo quando o acesso é feito é amplamente percebida pelos sujeitos na razão de prevalência bruta.

Contudo, quando se observa a análise da razão de prevalência ajustada, a percepção de FOMO quando não se checa redes sociais tem resultados significativos quando da afirmação positiva dos sujeitos, isto é, estes sujeitos apresentam 2,09\% de chances a mais de prevalência de alto FOMO. Observa-se, nesse sentido, que a percepção de FOMO aqui se descola da possibilidade de acesso, podendo ser vivenciada quando supostamente sua necessidade seria saciada.

É possível caracterizar o FOMO como um fenômeno partícipe da vida digital, e sua organização com reflexos comportamentais difusos e cujos objetos, efeitos e causas extrapolam a conjuntura dos dispositivos, serviços e plataformas da internet (FONSECA et al., 2018). Entende-se que o quadro derive dos mecanismos de recepção, reação e decisão sobre o conteúdo consumido e compartilhado, com reflexos sobre instâncias 
off-line (MANNO; ROSA, 2018). Isto é, o desencadeamento do FOMO, apesar de radicado no esteio on-line, expande-se para vivências do cotidiano sem a mediação como elemento detonador ou detrator.

Nas instâncias da escala de percepção de FOMO (ABEL; BUFF; BURR, 2016), a variável de sensação de que se está perdendo alguma coisa encontrou significância. FOMO percebido quando se está trabalhando também encontrou significância nas respostas que evidenciam frequência da sensação. As demais variáveis (FOMO durante as aulas, quando se está acompanhado e impossibilidade de acesso a redes sociais), embora significativos, foram demarcados com frequência relativa pelos sujeitos.

A prevalência de alto FOMO no grupo amostral aliado ao contexto supracitado pode gerar reflexões acerca da ocasião em que o fenômeno se torna factualmente percebido pelos sujeitos, com base nos dados e na literatura. Sem companhia de pares, o sentimento de perda é aumentado. A condição de se estar só ou a fuga à solidão parece um componente relacional e cotidiano a classificar as relações, tendo o uso das redes sociais como mecanismos de aplacar a sensação de solidão (BUDNICK; ROGERS; BARBER, 2020; MCDERMOTT, 2017).

A percepção frequente de FOMO no trabalho, por outro lado, acrescenta que a sensação está ligada a uma ansiedade por um objeto de novidade e satisfação que não se concebe ou se sabe precisar. Enquanto a solidão, ou a privação de atividades coletivas, implique em um quadro de perda de determinadas experiências por não participação em estado de frustração, o FOMO no trabalho acentua que o fenômeno provém de um desejo de urgência e de uma experiência inominável.

Quanto às dinâmicas produzidas pela percepção de FOMO no ambiente de trabalho, os resultados encontram ressonância em estudos que destacam o potencial dessa experiência em se mesclar à falta de motivação, ansiedade, condutas depressivas e desarmonia com as funções exercidas e o bem-estar do trabalhador (CASALE; FIORAVANTI, 2020; HUNT; MARX; LIPSON; YOUNG, 2018).

É recorrente, em consonância aos achados, que as dinâmicas de FOMO estão associadas a comportamentos de isolamento, depressão, solidão e ansiedade em ambientes distintos e associados ao uso ou gerenciamentos das potencialidades das redes sociais e das informações produzidas e consumidas nelas e por meio delas (KARGIN; POLAT; SIMSEK; 2020; LOPES; PÊGO; SANTOS, 2019; LOPES; SANTOS; PÊGO, 2019).

Acerca da associação de FOMO ao uso de determinados serviços de mídias digitais, não foram encontrados resultados significativos para as plataformas Instagram, Twitter, Facebook e Telegram. Entretanto, estudos demonstram que tais serviços estão associados a um quadro sócio-comportamental detonador de ansiedade, depressão, melancolia, queda de autoestima, agressividade, melancolia e neuroticismo (MORAHANMARTIN, 2009; ABELL; BUGLASS; BETTS, 2019; COCO et al., 2020; MILYAVSKAYA; SAFFRAN; HOPE; KOESTNER, 2018; RIFKIN; CINDY; KAHN, 2015). A configuração de consumo, visualização e compartilhamento de informações, bem como a tipologia de conteúdos, está associada ao acionamento de padrões detratores de personalidade e sociabilidade.

Observaram-se, porém, resultados significativos para usuários de WhatsApp e Messenger com frequência maior do que 5 vezes ao dia. Ambas as plataformas são sistemas de troca rápida de mensagens e, por sua interface e usabilidade prática, o uso massivo e amplo ocorre. Os dois serviços têm o uso atrelado a exercícios cotidianos diversos como estudos, trabalho e relações sociais com o agravante de que, por sua pervasividade, carregam consigo seus itinerários, ampliando os expedientes de trabalho e estudos, transpondo-os para o itinerário pessoal.

Esse quadro de uso irrestrito e por extensão dos meios os torna veículos estressores e dilatadores de outras experiências como sensações de urgência, irritabilidade, precarização do sono e do repouso, diminuição dos momentos de recreação e ócio, entre outras sensações de entristecimento e frustração. Há 
achados que coadunam as dinâmicas de funcionamento e uso desses serviços à potencialização de atitudes agressivas (COCO et al., 2020; MILYAVSKAYA; SAFFRAN; HOPE; KOESTNER, 2018).

As dinâmicas de consumo de informações nas timelines e nas funcionalidades das redes sociais e suas plataformas têm associação com a geração de FOMO e sua prevalência, bem como os efeitos comportamentais relatados (FABRIS; MARENGO; LONGOBARDI; SETTANNI; 2020; BALTA; EMIRTEKIN; KIRCABURUN; GRIFFITHS, 2018; DEMPSEY; O’BRIEN; TIAMIYU; ELHAI, 2019). Os fluxos de informação e a cadeia de comunicação, aliada aos tempos vertiginosos e virtualizados, produzem percepções aceleradas das fases e cadências do cotidiano, amplificando juízos, estereótipos e desejos.

A sensação de estar perdendo alguma coisa, uma atividade, um benefício ou uma experiência social gratificante, está associada a um anelo ansioso amplificado pela dinâmica de produção e consumo de informações contemporâneas, em que a internet, mais especificamente as redes sociais, tem esteio (BUGLASS; BINDER; BETTS; UNDERWOOD, 2017). Uma publicação, um feed ou uma timeline indicam uma vitrine de experiências para serem consumidas e não apenas lidas ou contempladas.

O consumo de conteúdo digital faz desejar experiências cada vez mais significativas para abastecer os fluxos informativos que as plataformas oferecem (BUGLASS; BINDER; BETTS; UNDERWOOD, 2017; REZENDE, 2014). A qualidade das experiências parece estar associada à qualidade de sua narrativa nas dinâmicas das mídias sociais. O compartilhamento e sua estética é que passam a deter o capital social e cultural, e não as atividades em si. Esse mecanismo faz aspirar e ansiar em um ciclo de consumo, produção e adequação (BUGLASS; BINDER; BETTS; UNDERWOOD, 2017; REZENDE, 2014).

O FOMO, nesse sentido, incorre como uma sensação exacerbada por anelo que não encontra objeto de satisfação definido, mas se torna aquiescente nas dinâmicas de produção, consumo e compartilhamento de informações de atividades sociais (ALT, 2018; FONSECA et al., 2018; MANNO; ROSA, 2018; MAFFESOLI, 2003). Performances são produzidas e signos de experiências coletivas são difundidos, introjetando sensações ansiosas de perda de recompensas e vivências sociais gratificantes quando as ocasiões são outras (trabalho, estudos, impossibilidade de acessar redes sociais e momentos sozinho) e até quando se está em situações de socialização.

Ao sabor de uma torrente de informações, imagens e interações, os sujeitos passam a construir um modus operandi para contingenciar sua experiência de vida ao fluxo que recebe do âmbito digital, criando laços. Estes laços formulam redes e emaranhados subjetivos cada vez mais densos, com os quais não se relaciona na posição de um jogador ou de um usuário experimental e, portanto, neutro (HOLLANDA, 2009).

Esse processo implica que os sujeitos manufaturem estes laços e redes em conexões cada vez mais atreladas à sua experiência de vida, em que os conteúdos pessoais, como os afetos, passam a impregnar suas performances (LANIER, 2018). De outro modo, a linguagem não é manifesta de modo único e controlado, mas se abre nas possibilidades de canalização e descarga (REZENDE, 2014). E, nesse ínterim, o FOMO se arrola.

Em um cenário em que a vida social tem se atrelado cada vez mais a uma experiência em rede e servida sobre telas, plataformas e aplicações, interessa compreender as dinâmicas mais profundas presentes nestas narrativas. A economia do compartilhamento de afetos torna-se tão abissal e rica quanto a economia de compartilhamento de informação, visto que o indivíduo o é inteiro em suas performances.

Uma vez caracterizado como um sentimento de avidez e estímulo exacerbado na consulta das redes sociais para o monitoramento de objetos indeterminados sob os quais se sente perder ou estar perdendo, o FOMO implica em uma perturbação da sociabilidade e do comportamento. A avaliação de sua prevalência, bem como suas correlações causais, resulta em um exame da qualidade de vida e do bem-estar.

Em um contexto em que dispositivos, acessos, conexões, serviços e redes se ampliam, convém monitorar os impactos desses processos sobre a atividade humana individual e coletiva. Entender as tessituras da 
sociabilidade digital pelo FOMO é tão abissal e rico quanto a economia de compartilhamento de informação atrelado à cibercultura, visto que o indivíduo é inteiro em suas performances.

\section{CONCLUSÃO}

O estudo revelou prevalência de alto FOMO entre os estudantes avaliados, sendo mulheres o grupo mais suscetível, bem como universitários na primeira metade de seus cursos. Há um número prevalente de estudantes que experimentam FOMO quando não podem acessar redes sociais por alguma razão, tendo aqueles que acessam WhatsApp e Facebook Messenger mais de 5 vezes por dia mais suscetíveis a alto FOMO.

FOMO também foi percebido nos sujeitos participantes em dois tipos de ocasiões, como o trabalho e quando se está sozinho. Resultados que encontraram ressonância em outros estudos, sugerindo que a percepção de FOMO é uma condição manifesta dos sujeitos inscritos sob uma sociabilidade de produção, consumo e compartilhamento de informações on-line.

O estudo se limitou a apenas uma IES privada de uma cidade proeminente do Norte de Minas Gerais. Embora se trate de um educandário expressivo, a investigação não contemplou outras IES públicas e privadas para um dado prevalente panorâmico. Sugere-se para estudos futuros, no mesmo contexto geográfico ou em outros, a ampliação do grupo amostral, de modo que a parcela universitária seja compreendida amplamente.

Observa-se por meio dos resultados que o alto FOMO está atrelado ao cotidiano e sua produção, interferindo nas relações e nas reações dos sujeitos. Desse modo, a sensação de que se perde uma experiência ou algum objeto de anelo não identificado acaba por remontar a um quadro ansioso, com precedente para a precarização das relações de sociabilidade, de estudos e de trabalho.

Na perspectiva de estudantes universitários, o FOMO pode se tornar um fator detrator de atenção e prejudicial ao desempenho, uma vez que conforme os dados, a sensação incorre do acesso às rede sociais, mas se desprende delas, afetando momentos em que esse acesso não é possível ou perdurando mesmo quando esse acesso é possível e plausível. Conforme os dados, o fenômeno é mais observável em ingressantes, o que pode indicar - a confirmar por estudos futuros - que novas gerações mais imersas no cotidiano digital sofram mais as consequências do contexto em questão.

Avaliar o FOMO, seja sua prevalência em determinados grupos sociais, seja por minúcias de sua percepção, seja por suas implicações em quadros clínicos de saúde, faz-se necessário a fim de mapear uma conjuntura de saúde e bem-estar imiscuída do substrato digital. Tecnologia, informação e comunicação são mais do que interfaces, mas terrenos nos quais o exercício do cotidiano se estabelece e no qual o devir humano - e, portanto, seu desenvolvimento, qualidade de vida e bem-estar - ocorrem.

\section{REFERÊNCIAS}

ABEL, Jessica P.; BUFF, Cheryl L.; BURR, Sarah A. View of social media and the fear of missing out: scale development and assessment. Journal of Business \& Economics Research, [s. I.], v. 14, n. 1, p. 33-44, 2016. DOI: https://doi.org/10.19030/jber.v14i1.9554. Disponível em: https://clutejournals.com/index.php/JBER/ article/view/9554. Acesso em: 26 abr. 2020.

ABELL, Loren; BUGLASS, Sarah L.; BETTS, Lucy R. Fear of missing out and relational aggression on Facebook. Cyberpsychology, Behavior, and Social Networking, [s. I.], v. 22, n. 12, p. 799803, 2019. DOI: https://doi.org/10.1089/cyber.2019.0071. Disponível em: https://pubmed.ncbi.nlm.nih. gov/31657626/\#: :text=Serial\%20mediation\%20analysis\%20demonstrated $\% 20$ that, rates $\% 20$ of $\% 20$ Facebook\%20relational\%20aggression. Acesso em: 26 abr. 2020. 
ALT, Dorit. Students' wellbeing, fear of missing out, and social media engagement for leisure in higher education learning environments. Current Psychology, [s. I.], v. 37, n. 1 128-138, 2018. DOI: https://doi. org/10.1007/s12144-016-9496-1. Disponível em: https://link.springer.com/article/10.1007/s12144-016-9496-1. Acesso em: 26 abr. 2020.

BALTA, Sabah; EMIRTEKIN, Emrah; KIRCABURUN, Kagan; GRIFFITHS, Mark D. Neuroticism, trait Fear of Missing Out, and phubbing: the mediating role of state Fear of Missing Out and problematic Instagram use. International Journal of Mental Health and Addiction, [s. I.], v. 18, p. 628-638, 2018. DOI: https://doi.org/10.1007/s11469-018-9959-8. Disponível em: https://link.springer.com/ article/10.1007\%2Fs11469-018-9959-8. Acesso em: 26 abr. 2020.

BAUDRILLARD, Jean. Simulacros e simulação. Lisboa: Relógio D’Água, 1991.

BAUDRILLARD, Jean. Tela total: mito-ironias do virtual e da imagem. 11. ed. Porto Alegre: Sulina, 2011.

BAUMAN, Zygmunt. Modernidade líquida. Rio de Janeiro: Zahar, 2001.

BRAGA, José Luiz. Uma conversa sobre dispositivos. Belo Horizonte: UFMG, 2020.

BUDNICK, Christopher J; ROGERS, Arielle P.; BARBER, Larissa K. The fear of missing out at work: examining costs and benefits to employee health and motivation. Computers in Human Behavior, [s. I.], v. 104, p. 106161, 2020. DOI: https://doi.org/10.1016/j.chb.2019.106161. Disponível em: https://www.sciencedirect.com/ science/article/abs/pii/S0747563219303735. Acesso em: 26 abr. 2020.

BUGLASS, Sarah L.; BINDER, Jens F.; BETTS, Lucy R.; UNDERWOOD, Jean D. M. Motivators of online vulnerability: the impact of social network site use and FOMO. Computers in Human Behavior, [s. I.], v. 66, p. 248-255, 2017. DOI: https://doi.org/10.1016/j.chb.2016.09.055. Disponível: https://www.sciencedirect.com/ science/article/abs/pii/S0747563216306902. Acesso em: 26 abr. 2020.

CASALE, Silvia; FIORAVANTI, Giulia. Factor structure and psychometric properties of the Italian version of the fear of missing out scale in emerging adults and adolescents. Addictive Behaviors, [s. I.] v. 102, p. 3072, 2020. DOI: https://doi.org/10.1016/.addbeh.2019.106179. Disponível em: https://www.sciencedirect.com/ science/article/abs/pii/S0306460319310834. Acesso em: 26 abr. 2020.

CASTELLS, Manuel. O poder da comunicação. São Paulo: Paz \& Terra, 2006.

CASTELLS, Manuel. O poder da identidade. 8. ed. São Paulo: Paz e Terra, 2013.

COCO, Gianluca Lo et al. Examining bi-directionality between fear of missing out and problematic smartphone use: a two-wave panel study among adolescents. Addictive Behaviors, [s. I.], v. 106,

n. 1, p. 43-69, 2020. DOI: https://doi.org/10.1016/j.addbeh.2020.106360. Disponível em: https://www. sciencedirect.com/science/article/pii/S030646032030099X. Acesso em: 26 abr. 2020.

DEMPSEY, Abigail E.; O'BRIEN, Kelsey D.; TIAMIYU, Mojisola F.; ELHAI, Jon D. Fear of missing out (FoMO) and rumination mediate relations between social anxiety and problematic Facebook use. Addictive Behaviors Reports, [s. I.], v. 9, p. 100150, 2019. DOI: https://doi.org/10.1016/j.abrep.2018.100150. Disponível em: https:// www.sciencedirect.com/science/article/pii/S235285321830169X. Acesso em: 26 abr. 2020.

FABRIS, Matteo Angelo; MARENGO, Davide; LONGOBARDI, Claudio; SETTANNI, Michele. Investigating the links between fear of missing out, social media addiction, and emotional symptoms in adolescence: the role of stress associated with neglect and negative reactions on social media. Addictive Behaviors, [s. I.], v. 106, p. 106364, 2020. DOI: https://doi.org/10.1016/j.addbeh.2020.106364. Disponível em; https://www.sciencedirect. com/science/article/abs/pii/S0306460319314133. Acesso em: 26 abr. 2020.

FALLOWS, Deborah. How women and men use the internet. Pew Research Center, [s. I.]: Pew Research Center, 2005. Disponivel em https://www.pewresearch.org/internet/2005/12/28/how-women-and-men-use-theinternet/. Acesso em: 14 ago. 2020. 
FONSÊCA, Patrícia Nunes da et al. Uso de redes sociais e solidão: evidências psicométricas de escalas. Arquivos Brasileiros de Psicologia, Rio de Janeiro, v. 70, n. 3, p. 198-212, 2018. Disponível em: http:// pepsic.bvsalud.org/scielo.php?script=sci_arttext\&pid=S1809-52672018000300014\&lng=pt\&nrm=iso. Acesso em: 26 abr. 2020.

GONÇALVES, Jaqueline Teixeira Teles; SILVEIRA, Marise Fagundes; CAMPOS, Maria Cecília Campos; COSTA, Lúcia Helena Rodrigues. Overweight and obesity and factors associated with menopause. Ciência \& Saúde Coletiva, Rio de Janeiro, v. 21, n. 4, p. 1145-1156, 2015. DOI: https://doi.org/10.1590/141381232015214.16552015. Disponível em: https://www.scielo.br/scielo.php?script=sci_arttext\&pid=S141381232016000401145\&Ing=en\&nrm=iso\&tlng=en. Acesso em: 26 abr. 2020.

HAN, Byung-Chul. No exame: perspectivas do digital. Petrópolis: Vozes, 2018.

HETZ, Patricia R.; DAWSON, Christi L.; CULLEN, Theresa A. Social media use and the fear of missing out (FoMO) while studying abroad. Journal of Research on Technology in Education, [s. I.], v. 47, n. 4, p. 259272, 2015. DOI: https://doi.org/10.1080/15391523.2015.1080585. Disponível em: https://www.tandfonline.com/ doi/abs/10.1080/15391523.2015.1080585?journalCode=uirt2. Acesso em: 26 abr. 2020.

HOLLANDA, Bernardo Borges Buarque de. Futebol, arte e política: a catarse e seus efeitos na representação do torcedor. Organização e Sociedade, Salvador, v. 15, n. 48, p. 123-140, 2009. Disponível em: https:/l periodicos.ufba.br/index.php/revistaoes/article/view/11011. Acesso em: 26 abr. 2020.

HUNT, Melissa G.; MARX, Rachel; LIPSON, Courtney; YOUNG, Jordyn. No more FOMO: limiting social media decreases loneliness and depression. Journal of Social and Clinical Psychology, Nova lorque, v. 37, n. 10, p. 751-768, 2018. DOI: https://doi.org/10.1521/jscp.2018.37.10.751. Disponível em: https://guilfordjournals. com/doi/abs/10.1521/jscp.2018.37.10.751. Acesso em: 26 abr. 2020.

JACKSON, Linda A.; ERVIN, Kelly S.; GARDNER, Phillip D.; SCHMITT, Neal. Gender and the internet: women communication and men searching. Sex roles, [s. I.], n. 44, p. 363-379, 2001. DOI: https://doi. org/10.1023/A:1010937901821. Disponível em: https://link.springer.com/article/10.1023/A:1010937901821. Acesso em: 26 abr. 2020.

JENKINS, Henry. Cultura de convergência. São Paulo: Aleph, 2009.

KARGIN, Maral; POLAT, Hilal Türkben; SIMSEK, Didem Coskun. Evaluation of internet addiction and fear of missing out among nursing students. Perspectives in Psychiatric Care, [s. I.], v. 56, n. 3, 726-731, 2020. DOI: https://doi.org/10.1111/ppc.12488. Disponível em: https://onlinelibrary.wiley.com/doi/abs/10.1111/ppc.12488. Acesso em: 26 abr. 2020.

LANIER, Jaron. Dez argumentos para você deletar agora suas redes sociais. São Paulo: Intrínseca, 2018.

LEMOS, Andre. Isso (não) é muito Black Mirror: passado, presente e futuro das tecnologias da informação e comunicação. Salvador: Edufba, 2018.

LIPOVETSKY, Gilles. Os tempos hipermodernos. São Paulo: Edições 70, 2013.

LOPES, Beatriz Calixto; PÊGO, Beatriz Ferraz; SANTOS, Gustavo Souza. Construindo o conceito de Fear of missing out: definições de FOMO nas Ciências Humanas e Sociais Aplicadas. In: SIMPÓSIO DE PESQUISA CIENTÍFICA UNIFIPMOC, 1., 19-22 jun. 2019, Montes Claros. Anais [...]. Montes Claros: UNIFIPMoc; 2019. p. 10.

LOPES, Beatriz Calixto; SANTOS, Gustavo Souza; PÊGO, Beatriz Ferraz. Vida digital, bem-estar e contemporaneidade: prevalência de Fear of Missing Out e propostas de intervenção. In: SIMPÓSIO DE EXTENSÃO UNIFIPMOC, 1., 28 nov. 2019, Montes Claros. Anais [...]. Montes Claros: UNIFIPMoc; 2019. p. 23.

MAFFESOLI, Michel. Mediações simbólicas: a imagem como vínculo social. In: MARTINS, Francisco Menezes; SILVA, Juremir Machado da. Para navegar no século XXI: tecnologias do imaginário e cibercultura. 3 ed. Porto Alegre: Sulina: Edipucrs, 2003. p. 37-48. 
MANNO, Maria Vittoria Maffei; ROSA, Carlos Mendes. Dependência da internet: sinal de solidão e inadequação social?. Polêmica, Rio de Janeiro, v. 18, n. 2, p. 119-132, 2018. DOI: https://doi.org/10.12957/ polemica.2018.37793. Disponível em: https://www.e-publicacoes.uerj.br/index.php/polemica/article/view/37793. Acesso em: 26 abr. 2020.

MCDERMOTT, Rachel. FoMO and the image of the self: from college campuses to Madison Avenue. 2017. 96 f. Trabalho de conclusão de curso (Bachelor of Arts) - Union College, Schenectady, 2017.

MILYAVSKAYA, Marina; SAFFRAN, Mark; HOPE, Nora; KOESTNER, Richard. Fear of missing out: prevalence, dynamics, and consequences of experiencing FOMO. Motivation and Emotion, [s. I.], v. 42, p. 725-737, 2018. Disponível em: https://link.springer.com/article/10.1007/s11031-018-9683-5. Acesso em: 26 abr. 2020.

MORAHAN-MARTIN, Janet. The gender gap in the internet use: why men use the internet more than women a literature review. CyberPsychology \& Behavior, [s. I.], v. 1, n. 1, p. 3-10, 2009. DOI: https://psycnet.apa.org/ doi/10.1089/cpb.1998.1.3. Disponível em: https://psycnet.apa.org/record/2000-16536-001. Acesso em: 26 abr. 2020.

PRZYBYLSKI, Andrew K.; MURAYAMA, Kou; DEHAAN, Cody R.; GLADWELL, Valerie. Motivational, emotional, and behavioral correlates of fear of missing out. Computers in Human Behavior, [s. I.], v. 29, n. 4, p. 1841-1848, 2013. DOI: https://doi.org/10.1016/j.chb.2013.02.014. Disponível em: https://www.sciencedirect. com/science/article/abs/pii/S0747563213000800. Acesso em: 26 abr. 2020.

REZENDE, Renata. A catarse cotidiana: performances dramáticas no Facebook. Cultura Midiática, João Pessoa, v.13, n. 3, p. 142-156, 2014. Disponível em: https://periodicos.ufpb.br/index.php/cm/article/view/24498. Acesso em: 26 abr. 2020.

RIFKIN, Jacqueline; CINDY, Chan; KAHN, Barbara. FoMO: how the fear of missing out leads to missing out. North American Advances in Consumer Research, [s. I.],v. 43, p. 244-248, 2015. Disponível em: https:// www.acrwebsite.org/volumes/1019794/volumes/v43/NA-43. Acesso em: 26 abr. 2020.

VAUGHN, Jessica. JWT's Ann Mack delves into FOMO at SXSWi. Londres: JWT Marketing Communications. 2012. Disponível em: http://www.jwtintelligence.com/wp-content/uploads/2012/03/F_JWT FOMO-update 3.21.12.pdf. Acesso em: 12 fev. 2019. 